\title{
Using A New Endodontic Tooth Model as an Alternative in Clinical Education Course During the Covid-19 Pandemic
}

Sareh Said Yekta-Michael ( $\sim$ samichael@ukaachen.de)

RWTH Aachen University

Christoph Maria Färber

RWTH Aachen University

Alexander Heinzel

RWTH Aachen University

\section{Research Article}

Keywords: artificial tooth model (RCT), education, endodontics, feasibility study, physical properties

Posted Date: January 6th, 2021

DOI: https://doi.org/10.21203/rs.3.rs-134995/v1

License: (c) (i) This work is licensed under a Creative Commons Attribution 4.0 International License.

Read Full License 


\section{$1 \quad$ BMC medical education}

2 Using a new endodontic tooth model as an alternative in clinical education course during

3 the Covid-19 pandemic

4

5 Sareh Said Yekta-Michael ${ }^{1,2}$, Christoph Maria Färber ${ }^{3}$, Alexander Heinzel ${ }^{4}$

6

7 1: Department of Orthodontics, RWTH Aachen University, Aachen, Germany

8 2: Interdisciplinary Center for Clinical Research, RWTH Aachen University, Aachen,

9 Germany

3: Department for Operative Dentistry, Periodontology and Preventive Dentistry, RWTH

11 Aachen University, Aachen, Germany

4: Department of Nuclear Medicine, Medical Faculty, RWTH Aachen, Aachen, Germany

Running Title: New tooth model and endodontic education

Keywords: artificial tooth model (RCT); education; endodontics; feasibility study; physical properties

* Corresponding author:

Department of Conservative Dentistry, Periodontology and Preventive Dentistry, Interdisciplinary Center for Clinical Research, Aachen University, Pauwelsstr. 30, 52074

Aachen, Germany

Tel.: $004924180-8035247$

Fax: $004924180-82468$

Email: samichael@ukaachen.de 


\section{Abstract}

Background The COVID-19 pandemic massively impacts endodontic teaching, and a dramatically reduced number of patients is registered in clinical courses. This could be countered with suitable training aids. Based on treatment errors made by students in the last five years of endodontic courses at RWTH Aachen University (Germany), a new artificial root canal treatment model (DRSK RCT) was developed. The model was aimed to be radiopaque and to simulate the tactile feel during instrumentation in a realistic manner. Unlike already existing 3D-printed tooth replicas, the RCT has anatomical root canals with a narrow lumen with its width matching an ISO size 6 endodontic file.

Methodology 35 fourth-year students and seven dental demonstrators performed endodontic treatments on both the DRSK RCT and extracted teeth. Students and demonstrators answered a questionnaire on a scale ranging from 1 to 7 (poor to high) for different items (part 1). After the first study, changes in the materials and root canal anatomy were applied to the model. Then, the whole study was repeated and evaluated (part 2). Finally, it was evaluated whether the models could replace patient treatment during the Covid-19 pandemic.

Results Ratings by students and dental instructors $(5.1 \pm 0.4$ and $5.3 \pm 1.5$ [mean \pm SD], respectively) in the first study increased after modifications of the DRSK RCT $(5.5 \pm 0.5$ and $6.2 \pm 0.8$, respectively). Radiographs of the models were excellently assessable. The properties of the DRSK RCT were found to be realistic, thus allowing students to perform a satisfactory simulation of root canal treatment and being rated sufficient in substituting patient treatment during COVID-19 pandemic.

Conclusion The analysis suggests that the DRSK RCT has the ability to improve endodontic technique and education. Visible root canals enable students to observe the treatment process. All steps of a regular root canal treatment can be simulated. Further studies are needed to investigate the outcome of treating the first patient after practicing on the DRSK RCT. 


\section{Background}

60 Similar to any other field of knowledge, dentistry constantly embraces novel and innovative

61 modes of training that could potentially facilitate and improve the learning curve for new students. For endodontic education, attempts were made to establish guidelines for the undergraduate training in dental schools [1], which can ensure the achievement of a certain level of competence. To further improve the endodontic education, new forms of teaching methods are required. A study from the United Kingdom showed that teaching methods developed during the last decade led to a wider variation of methods [2]. Despite this, several studies point out that the quality of root canal treatments performed by undergraduate students is often not satisfactory $[3,4,5,6,7,8]$. Thereby, the way of teaching endodontics has a major influence on the quality of root canal treatments performed by students [9].

With regards to endodontics, the traditional preclinical training involved practicing the procedure on extracted natural teeth. Nevertheless, this practice was fraught with concerns over infection control and required disinfection of the teeth [10]. Some materials traditionally used for the purpose of efficient disinfection, such as formalin, proved to have hazardous effects of their own [11]. Furthermore, the supply of natural teeth is not infinite, and combined with the dwindling number of extracted intact teeth - probably as a result of improvement in health standards - it presents a problem for instructors and students in preclinical endodontics [12].

The COVID-19 pandemic emphasizes the importance of alternative teaching methods in dental education and both, students and instructors prefer these methods over having a non-semester [13]. Current protective measures have a massive impact on university teaching $[14,15]$. Especially the field of endodontics is affected, since teaching in clinical courses includes patient treatments that cannot be simply substituted by e-learning programs. Although safety concepts may not completely prohibit patient treatments, a very reduced number of patients is evident 
due to the pandemic. Effective training models that enable the implementation of a realistic root canal treatment, could adequately meet these challenges.

The simplest of the alternative endodontic training models come in the shape of endodontic blocks with a built-in conduit that approximates in its shape and diameter the root canals of natural teeth [16]. Because they do not represent the external anatomy of the crown and root, these endodontic blocks are of limited educational value. These models do not allow the students to learn how to avoid the procedural problems pertaining to the distance between the canal and external surface of the tooth and lateral or apical perforations of the roots.

Further advances in 3D printing technology promised more sophisticated models simulating a complete tooth including a hollow space representing the root canal system. Apart from their application in pre-clinical courses, these models can be also used in the context of researchers requiring a simulation of the internal anatomy of the teeth [17]. In recent years, different brands of such models have been brought to the market, in turn prompting researchers and academics to perform studies and investigate their properties and suitability as training tools, thus replacing the extracted teeth. Another study found artificial tooth models suitable for endodontic training [18]. Nevertheless, the results of these studies suggest that complete replacement of natural teeth with artificial teeth for endodontic training should be regarded with caution. The physical properties of the models have been of special concern to preclinical endodontics instructors. To be suitable for the desired learning experience, these models are expected to feature physical properties as similar as possible to those of a natural tooth. However, studies showed that, despite advantages of artificial teeth, their physical characteristics are not yet completely satisfactory $[19,20]$. In case of one particular model made from a hydroxyapatite-based matrix, a study showed that it is similar to a natural tooth in regard to many physical properties [21]. This study focused exclusively on the physical properties of the model. It did not provide any 
107 information on the actual experience of practicing root canal treatment on synthetic teeth made 108 from this material.

109 Finally, a recent study introduced the concept of 3D-printed replicas of extracted teeth for 110 endodontic training [22] and recommended it as a practice that dental schools have to embrace. 111 In this study, the researchers produced exact copies of natural teeth and measured their 112 properties and accuracy. However, there remain limitations associated with this concept, and 113 therefore there is a continued demand for commercially produced models. Any modification of 114 the model design, including shape, curvature, length, and width of the canals, falls beyond the 115 scope of the proposed simple reproduction of a micro-computed tomography (micro-CT) file. 116 It can only be achieved by employing computer-aided design software applications, which 117 owing to their sophistication - normally necessitates enlisting the help of expert personnel with 118 computer design skills.

119 The present study aimed to evaluate the artificial root canal treatment model (DRSK RCT), 120 which is based on errors made by students in the last 5 years. In contrast to already existing 3D121 printed tooth replicas, the DRSK RCT aims to have root canals with a narrow lumen with its 122 width matching an ISO size 6 endodontic file so as to enable students to negotiate the root canal 123 system with endodontic scouting files. We evaluated whether the DRSK RCT is radiographable 124 and also to what extent it permits practicing various shaping and cleaning techniques. In 125 addition to measuring the physical properties of this model, the study aimed to assess the 126 subjective experience of the users (including both students and instructors). By questioning 127 participants about every stage of the root canal treatment performed on this model, the present 128 study evaluated the educational value of DRSK RCT from a practical standpoint. The 129 participating students and dental instructors assessed the DRSK RCT model with regard to its 130 physical properties and its suitability to realistically imitate an endodontic treatment, to 131 optimize the tooth model according to the results of the evaluation for endodontology courses. 
132 Finally, it was evaluated whether the models could replace patient treatment during the Covid-

13319 pandemic.

\section{Methods}

136 Thirty-five students who had successfully completed the sixth semester - and therefore had

137 knowledge of endodontics - and seven instructors participated in this study. The study was

138 performed in the dental faculty of RWTH Aachen University in Germany, after getting approval

139 of the internal ethics committee.

The Artificial Root Canal Treatment Model (DRSK RCT)

142 The model is manufactured by DRSK Group AB, Sweden

143 (https://drsk.com/pages/Training/RCT/root_canal_model_RCT.html). It comprises a model

144 designed and prepared in 3D with pulp cavity and root canal(s) that are considerably narrow in

145 dimensions and shape than their natural counterparts. The model is designed for practicing

146 every stage of a root canal treatment. Tooth models contain complete and intact crowns and

147 roots with a hollow space inside, simulating the pulp chamber and root canals. To achieve a

148 more realistic representation, the hollow space in DRSK RCT was filled with a soft red-colored

149 resin imitating the pulp.

150 The DRSK RCT used in this study was developed based on commonly seen errors made in the

151 last five years by students of endodontic courses in RWTH Aachen University in Germany

152 (Figure 1). 

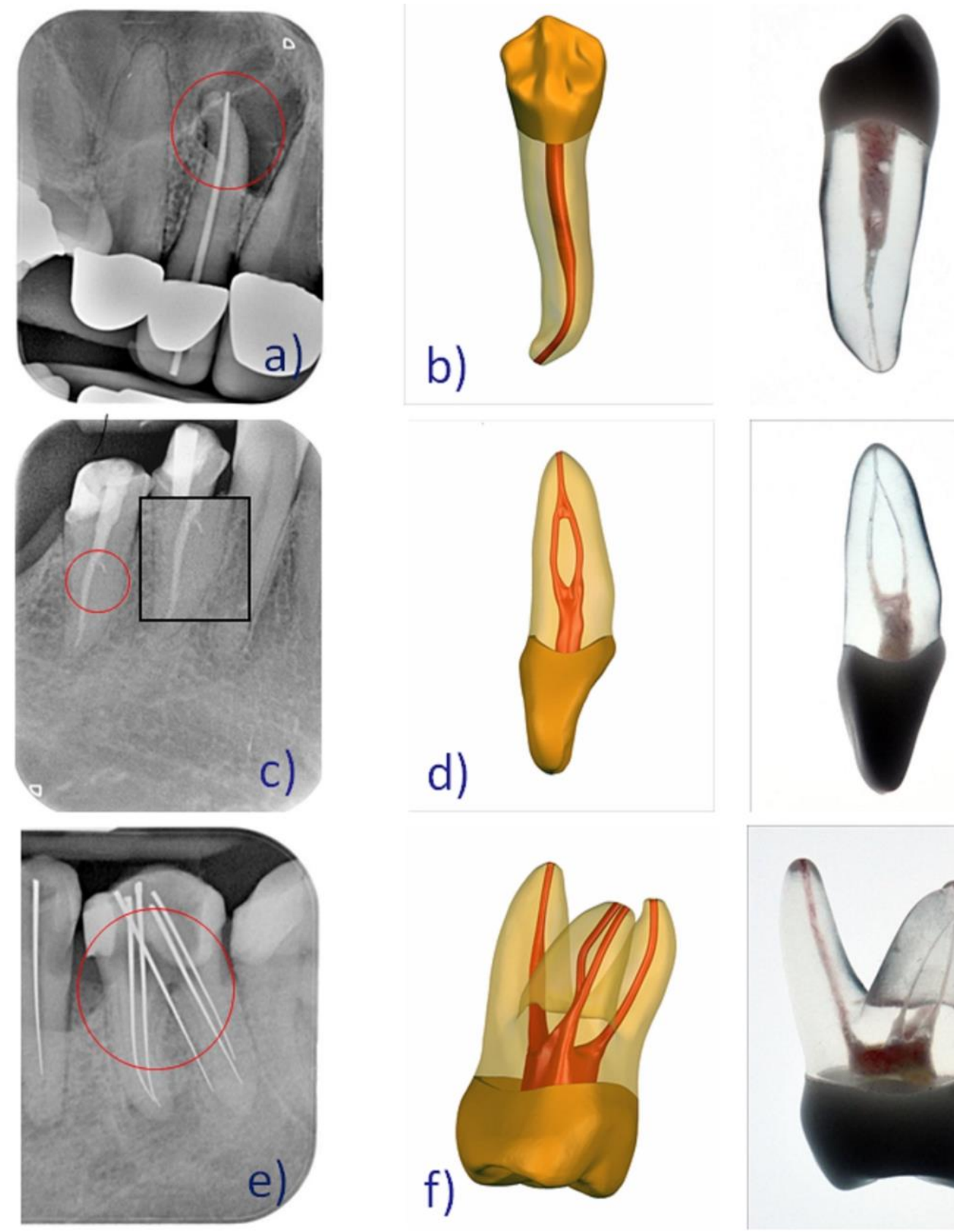

d)
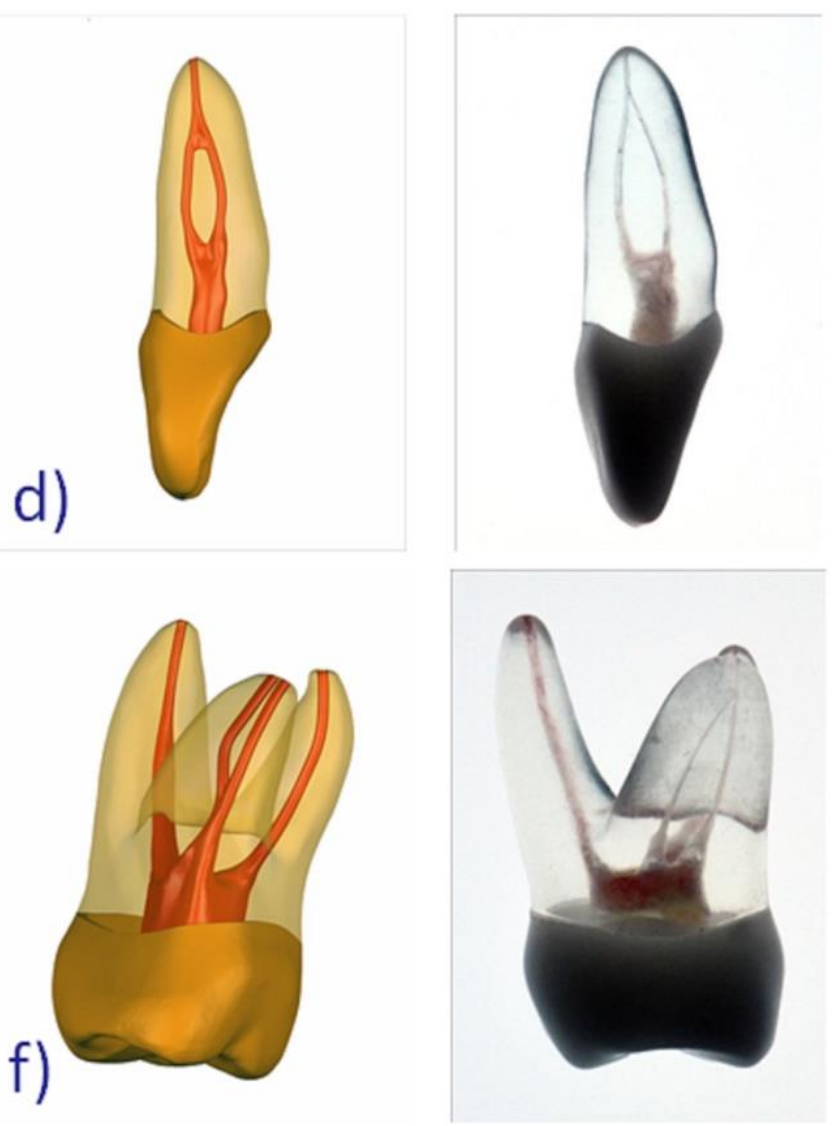

158

159

160 The roots of DRSK RCT were transparent, which permitted the observation of the progression

161 of the simulated treatment (Figure 2). 


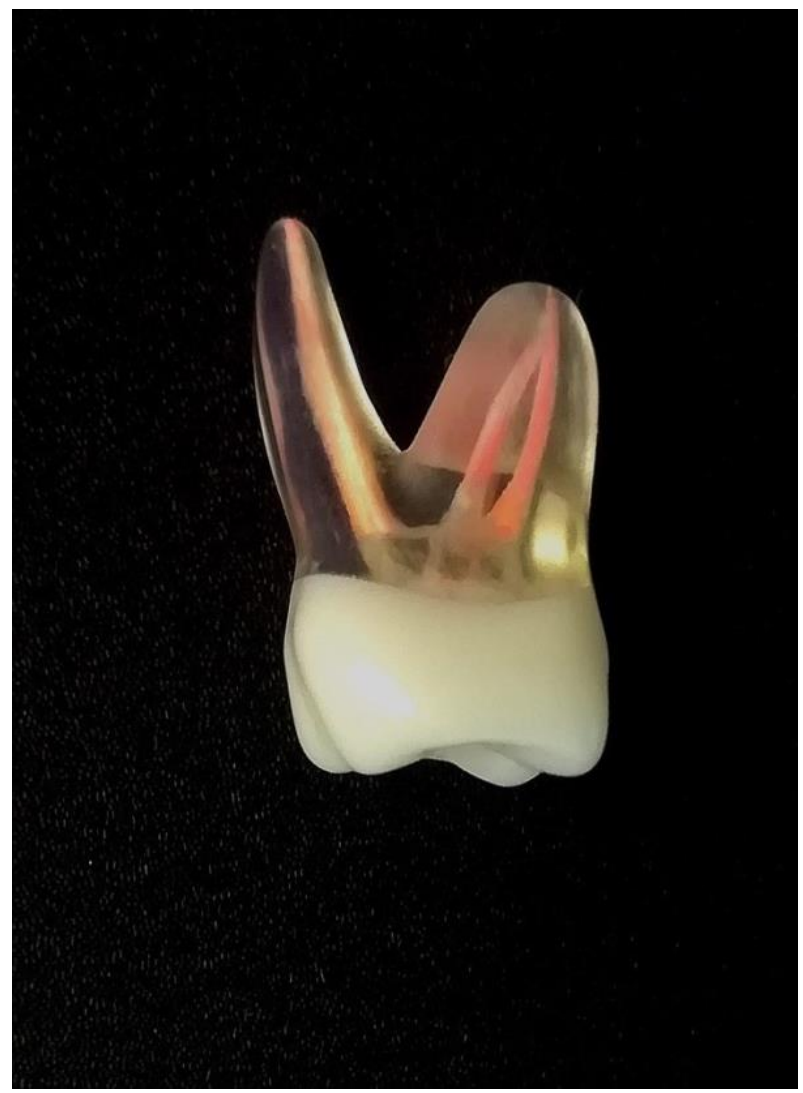

168 We developed three variations of tooth models, representing different types of teeth: incisors,

169 premolars, and molars. The incisor was represented by a model of a first maxillary incisor. A

170 hidden second root canal was designed as a special feature of this tooth model. Undetected root

171 canals often lead to failure of endodontic treatment. Inexperienced students often fail to detect

172 all existing root canals of a tooth, especially when the number or configuration of root canals

173 deviates from the norm. As an example of a premolar, a mandibular first premolar with a single

174 root canal was chosen. It has been observed that students tend to straighten root canals during

175 mechanical preparation by not bending the files used. The chosen anatomy of the premolar in

176 the DRSK RCT model makes it inevitable to carefully scout the root canal with a bent file to

177 preserve its anatomy and not be stuck in the apical third of the model root canal without entering

178 the apical constriction.

179 A maxillary first molar with three roots and four root canals was selected to demonstrate molars.

180 The molar model featured a second mesiobuccal root canal (MB2) corresponding to the 
established predominance of this root canal configuration for maxillary first molars [23]. While trying to locate the MB2, students - owing to their lack of experience - may cause perforations in the pulp chamber floor. Unsatisfactory anatomical skills lead to drilling in wrong places to locate the MB2. This tooth model will provide knowledge of the location of MB2 and assist in developing the skills to locate it. The study consisted of two parts, as described below.

\section{Part 1 (students)}

Before using DRSK RCT, all students practiced on extracted teeth. Then, the participants were asked to perform a routine root canal procedure on the DRSK RCT models, starting by drilling an access cavity with Endo-Z burs (Dentsply Sirona). A secondary access cavity was prepared using Mueller drills (Komet dental) and Gates-Glidden drills (Komet dental).

After taking the radiograph (Figure 3), hand files (K-Files, SybronEndo) were used for mechanical root canal preparation.

Figure 3

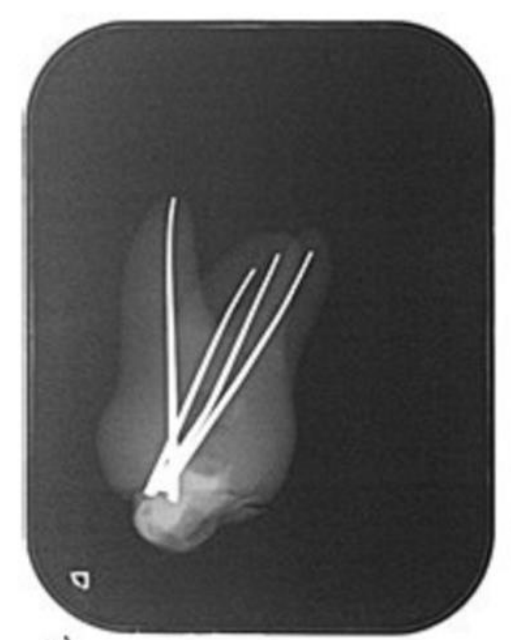

a)

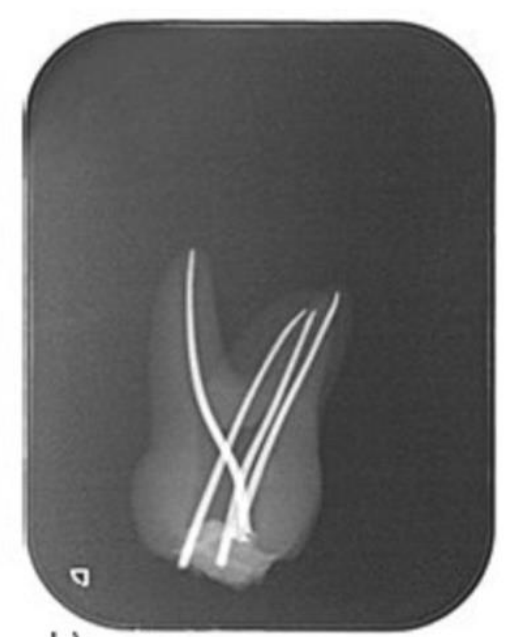

b)

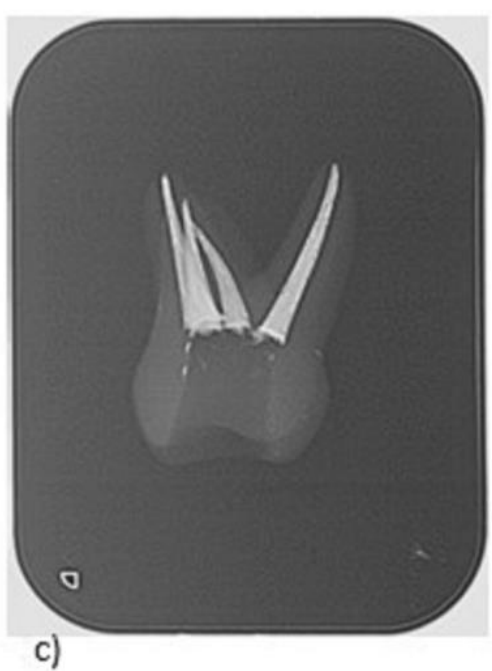

c)

Students enlarged the canals until an ISO 35 file could reach the working length. Students continued shaping the canals, using the step-back technique to flare them to the size of an ISO 60 file. Between instrumentation, canals were rinsed and irrigated with 3\% sodium hypochlorite solution, and patency was ensured by using ISO size 10 files. Radiographs were taken during 
the process in accordance with the routine protocol (once to determine the working length, then with the master cone, and the last one after obturation). For taking radiographs, the setting of the X-ray machine was changed to levels normally used for children. Canal obturation in this study was performed using gutta-percha (ANTAEOS GuttaPercha Points) with AH26 as the 204 sealer.

\section{Part 1 (instructors)}

Another cohort in this study consisted of seven instructors who performed the same procedure of simulated root canal treatment - as described for the student group - on the DRSK RCT model. After the procedure, all participants - students and instructors - received a questionnaire about their experience with the model. The questions were in a Likert-type scale format, and they had to be answered by ranking one aspect of the model from the scale of 1 to 7 . The higher values for each question indicated a better simulation of that particular aspect.

\section{Part 2 (modified model)}

This part of the study was conducted after evaluating the questionnaires answered by both

216 students and dental instructors. According to the evaluations the following modifications were made in the DRSK RCT on the basis of the findings:

Modifications of DRSK RCT: After modifications, the incisor's special feature was the canal morphology, containing a hidden second root canal corresponding to Vertucci type III canal configuration, with buccal and palatal canals merging together in the apical third. The mandibular first premolar single root canal became a J-shaped turn near the apex. The MB2 of the molar was extended. To increase the contrast between the model and gutta-percha, the composition of the material was modified. Furthermore, to improve the tactile feel of entering

224 the pulp of the endodontic model, the composition of the material was changed. Additionally, 225 root canal diameters were reduced for better representation of natural teeth. After the 
modifications were implemented, students performed root canal treatments on the new DRSK RCT. For the second part, the same questionnaire was used. In this manner, the differences in ratings could be analyzed.

\section{Evaluation of students' performances}

231 Finally, the seven dental instructors evaluated these simulated treatments. The success of the 232 students' performance was determined by the correct length and shape of the root canal fillings 233 as well as their homogeneity. The correct length was determined by the obturation ending 0.5 $2341 \mathrm{~mm}$ before the radiographic apex. All seven dental instructors blindly evaluated all treated DRSK RCT. Only tooth models with correct length, shape, and homogeneity of the root canal filling were rated as "positive" (Table 1).

\section{Statistical Analysis}

239 The accumulated data for the two sets of questionnaires was entered into an Excel spreadsheet 240 and means and standard deviations for each question were calculated. Paired t-tests were 241 performed to compare the data before and after modifications. The significance of differences 242 was determined at $\mathrm{p}<.05$. The software IBM® SPSS $®$ Statistics 22.0 (IBM®, USA) was used 243 for all statistical calculations. Graphics were created with Excel (Microsoft Office Excel $2442007 @)$.

\section{Results}

247 All radiographs of the DRSK RCT were clearly assessable; there was a sufficient contrast 248 between the model and gutta-percha. Moreover, the outlines of roots were completely visible. 249 For the first part of the study, student's ratings of the DRSK RCT ranged from 4.4 to 5.9 on a 
scale of 1 to 7 , with the mean of their ratings being $5.1 \pm 0.4$ (mean $\pm \mathrm{SD}$ ). For the second part

251 of the study performed after improving the RCT, students' ratings ranged from 4.6 to 6.1 . The mean of their ratings increased to $5.5 \pm 0.5$ (mean $\pm \mathrm{SD})$.

253 Students' ratings of the pulp anatomy, the ability of the model to simulate a natural tooth, ability 254 to flare canals, and preference for using the DRSK RCT in preclinical evaluations were 255 significantly higher $(\mathrm{p}<.05)$ for the improved DRSK RCT than for the model used before 256 modifications. The average rating of the pulp anatomy increased from $5.4 \pm 1.1$ for the first DRSK RCT to $5.9 \pm 0.9$ for the improved model. Likewise, the rating of the model's ability to simulate natural teeth increased from $4.6 \pm 1.3$ for the first DRSK RCT to $5.3 \pm 1.2$ for the modified DRSK RCT. Furthermore, students found that flaring of root canals could be 260 performed in a significantly better manner on the modified DRSK RCT; the ratings increased 261 from $4.8 \pm 1.6$ to $5.6 \pm 1.0$ after the material was modified. In the first part, the mean rating of the suitability of DRSK RCT as an evaluation tool in preclinical course was $5.0 \pm 1.7$, whereas after modifications, it increased to $5.9 \pm 1.1$ (Figure 4). 


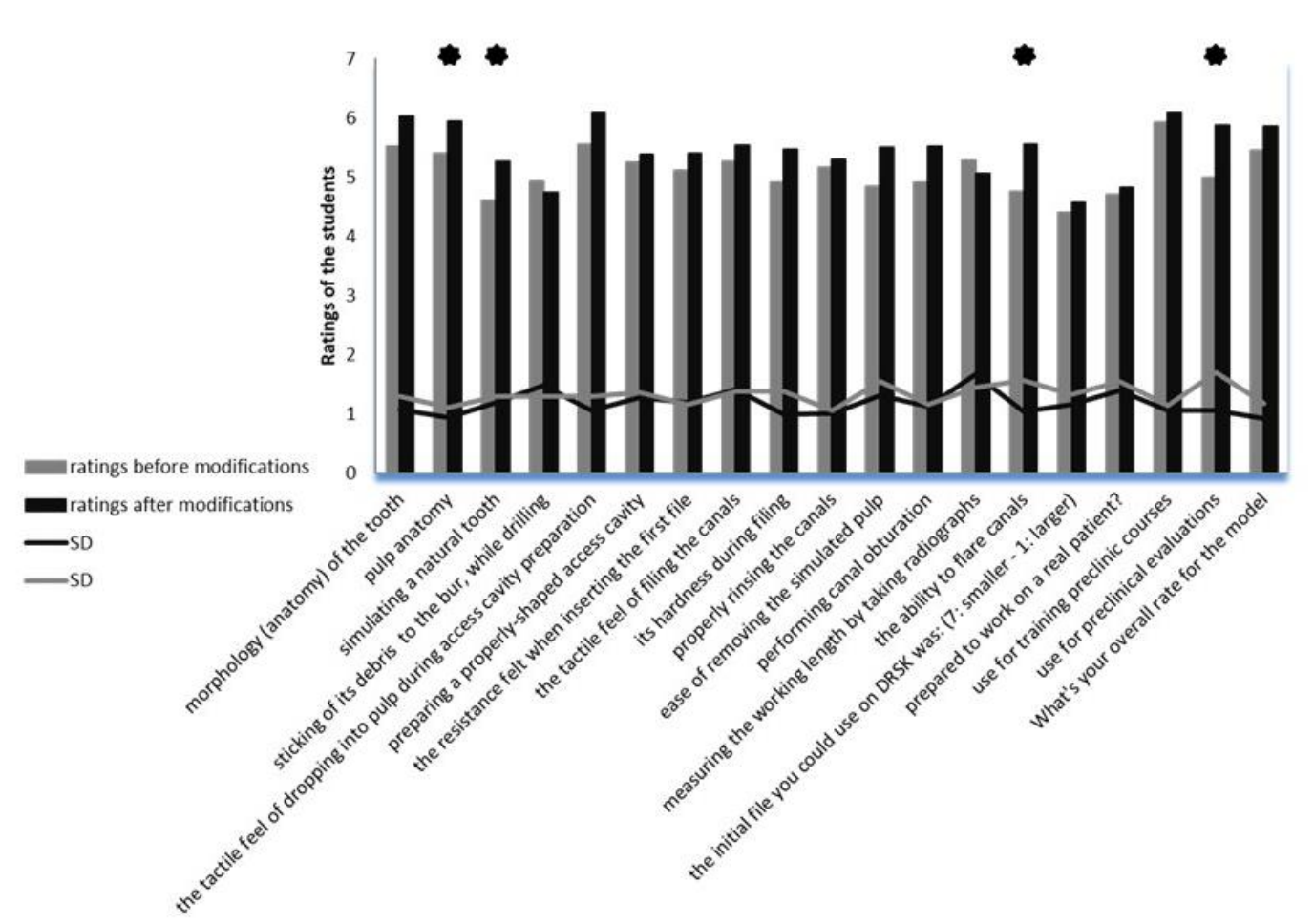

275 The mean ratings by dental instructors increased from $5.3 \pm 1.5$ to $6.2 \pm 0.8$ following model 276 improvement (Figure 5). The simulated hardness felt during instrumentation, in particular, was rated significantly higher $(\mathrm{p}<.05)$ after DRSK RCT modifications (from $5.0 \pm 0.7$ to $6.3 \pm 0.4$ ). When asked about the student's readiness to work on a real patient after practicing on RCT, the mean rating was $4.8 \pm 2.3$, whereas this rating increased to $6.6 \pm 0.7$ for the enhanced model (Figure 5). 


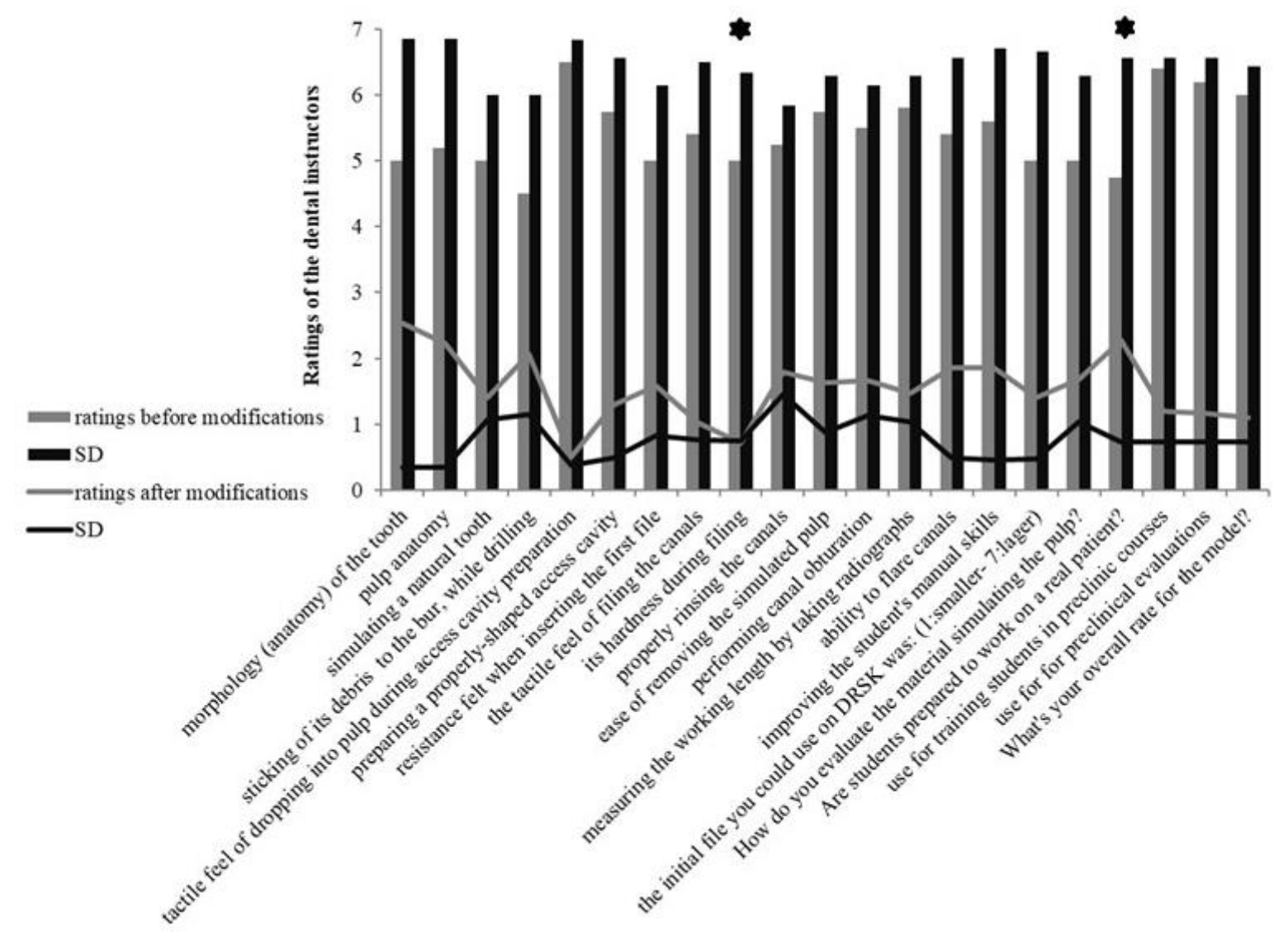

289 In light of the current COVID-19 pandemic, the same seven dental instructors rated the suitability of the DRSK RCT tooth model as a possible substitute for patient treatments at 5.6

$291 \pm 0.5$, in case that root canal treatment is not possible on a patient in the course due to the 292 pandemic.

293 Eventually, the dental instructors positively evaluated $87.35 \%$ of the students' performances on 294 the modified training aids, whereas accurate length and homogeneity of the root canal filling were the criteria for a positive rating. 
299 Table 1 Assessment of root canal treatments performed by students on the DRSK RCT tooth 300 model

\begin{tabular}{cccc}
$\begin{array}{c}\text { Dental Instructor } \\
(\mathrm{n}=7)\end{array}$ & $\begin{array}{c}\text { Students' Success Rate } \\
(\mathrm{n}=35)\end{array}$ & Successful & Not Successful \\
\hline Dental Instructor 1 & $88.57 \%$ & 31 & 4 \\
Dental Instructor 2 & $88.57 \%$ & 31 & 4 \\
Dental Instructor 3 & $85.71 \%$ & 30 & 5 \\
Dental Instructor 4 & $82.86 \%$ & 29 & 4 \\
Dental Instructor 5 & $88.57 \%$ & 31 & 4 \\
Dental Instructor 6 & $88.57 \%$ & 31 & 4
\end{tabular}

\section{Discussion}

303 In this study, we used an artificial root canal treatment model (DRSK RCT) with an 304 anatomically accurate design. According to its manufacturer, this model can facilitate practicing 305 various shaping and cleaning techniques. Instead of merely measuring the physical properties 306 of this model, the study evaluated the subjective experience of the users (including both students 307 and instructors). The DRSK RCT was designed virtually based on errors made in the last five 308 years by students of endodontic courses. In addition, the anatomy of extracted teeth was used 309 as an overall reference.

310 When drilling an access cavity, the material used for the tooth model must provide sufficient 311 resistance such that its difference from the material filling the pulp chamber can be perceived 312 clearly. Some authors already mentioned that resin used for endodontic tooth models does not 
match the hardness of dentine. Therefore, drilling an access cavity becomes more complicated

314 because the perceived difference in resistance is insufficient [20, 24, 25]. The hardness of

315 dentine-imitating resin and the accuracy of anatomical reproduction affect the degree of

316 difficulty of working with endodontic models in general [26]. Soft resin complicates its

317 distinction from the soft material imitating the pulp, whereas excessively hard resin leads to

318 canal blockage [20].

319 The DRSK RCT model allows a close simulation of performing endodontic treatment on a 320 natural tooth. This is evidenced by the good ratings achieved in our study. One major alteration 321 to the model involved changing the material used in the manufacturing of DRSK RCT. 322 Consequently, access drilling, root canal preparation, and obturation were performed more 323 easily because the use of harder material offers more resistance. When entering the pulp 324 chamber, the tactile feel is crucial for not harming the pulp floor by accident. This is important 325 for beginners, as they are not used to paying attention to the difference between dentine and the 326 hollow space of the pulp chamber. Sticky debris produced while shaping the root canals blocks 327 the canals and cannot be flushed out easily; however, the stickiness of the debris is reduced 328 when a harder material is used. A material with hardness similar to that of dentine and cement 329 of natural teeth accurately represents the tactile feel while performing root canal treatment on 330 patients, which is the actual reason for using endodontic training aids.

331 Students highly rated DRSK RCT for potential use in preclinical training, which may be 332 because each model has the exact same anatomy and therefore creates equal and fair conditions 333 for every student. However, there are no two natural teeth with the exact same anatomy. Owing 334 to the large variation observed in the anatomy of natural teeth, a further major alteration made 335 to the studied model included modifying the shapes of the root canals to create a more complex 336 and realistic root canal system. Considering that each tooth requires a unique mode of 337 endodontic intervention, any evaluation procedure based on the use of natural teeth would 
become complicated, and questions may be raised over its fairness. These difficulties emphasize

339 the need to seek alternative training models such as the DRSK RCT for practicing root canal

340 treatment during preclinical courses.

341 The ambition to have models with translucent roots has been on record since as early as 1975

342 [27]. To that end, different methods have been devised to increase the transparency of the model

343 by applying chemical agents. However, this is often accompanied by undesired effects such as

344 altered physical properties. In the present study, the transparent roots of the model may also

345 have influenced the students' opinion about the DRSK RCT as a training aid in preclinical

346 courses. Because endodontic treatments are performed inside root canals and obscured from

347 view, students often feel insecure when they are unable to see what they are doing. Making the

348 treatment procedure visible allows inexperienced students to gain a deeper understanding of the

349 process. If any error occurs, the cause can thus be quickly identified [21], which is essential for

350 the students' learning process. Such models may also benefit research and testing of certain

351 devices and equipment when it is required to have a direct view of the canals and observe how

352 the equipment functions inside.

353 In root canal treatments on previous training models, endodontic files of ISO size 15 were used

354 for scouting. Owing to technical considerations, printing tooth models for endodontic training 355 with optimal root canal diameter was challenging [22]. In a study that compared several 356 endodontic training aids, a model named TrueTooth by DELabs, built on the basis of micro-CT 357 scans of natural teeth, was favored owing to its anatomy and material properties being closer to 358 reality [26]. For instance, diameters of the MB2 of TrueTooth \#19 (02) are indicated to be 0.12 $359 \mathrm{~mm}$ (apical part) and $0.28 \mathrm{~mm}$ (coronal part) on its manufacturer's website. Because the initially 360 used file for root canal treatment in real patient is often of a significantly lower ISO size $(6,8$, 361 or 10), it is part of our department's protocol of root canal treatment to use c-pilot files of ISO 
size 6,8 , or 10 for scouting the root canal system. This needs to be practiced in preclinical training as well and thus requires models with very narrow root canals.

364 After modification, the DRSK RCT received higher ratings for most points from both cohorts.

365 Owing to the changes in the material, various aspect of simulated root canal treatment such as 366 the stickiness of debris, the feel of drilling the access cavity, or the perceived hardness during 367 filing were improved. Furthermore, radiographs of the tooth model were easier to read as the 368 material became more radiopaque.

The 3D print technology used for producing the DRSK RCT made it possible to change the shapes of the root canals and modify them as desired without incurring substantial costs. The similarity of the DRSK RCT to a natural tooth makes it an ideal training tool in endodontics.

372 The ratings by students and instructors alike point to the suitability of the studied model to be 373 used in endodontic courses. During the COVID-19 pandemic it could be advantageous to 374 incorporate this training aid into the clinical curriculum as supplying students with suitable 375 patients to perform endodontic treatments becomes challenging. Many patients currently avoid 376 the student course out of fear of infection and postpone treatments. In addition, as the number 377 of cases increases, it is becoming more common for patients to be quarantined, making it 378 impossible to carry out treatment properly. As many universities exclusively train students on 379 phantom heads during the current pandemic the tooth model would perfectly fit into their 380 curriculum. Both, students and tutors rated the model as suitable for training and evaluation in 381 preclinical courses and students also felt well prepared for their first root canal treatment on 382 patients. The realistic anatomy and properties of the model allow students to learn the 383 difficulties of endodontic treatments and enable them to practice any clinical step of the 384 procedure. Ratings by the dental instructors indicate its potential use in clinical courses. 385 Reduced patient numbers or concerns about the safety of patients, students and staff in the face 386 of high infection rates might force universities to completely substitute patient treatments with 
the work on phantom heads or models [28]. Even though training on models can never replace training on patients, it is probably the best alternative given the current circumstances.

In the last part of the study, seven dental instructors evaluated the results of endodontic treatments performed on the DRSK RCT. The length of the gutta percha filling was taken as

391 the main measure of a successful treatment. The success rate ranged between $82.86 \%$ to $39288.57 \%$, indicating that the students were able to perform proper endodontic treatments on the 393 developed tooth model. As radiopacity is the key to determine the correct working length, it is 394 crucial that the apical third is clearly visible on radiographs, and thus the correct length of the 395 gutta percha filling can be perceived [26].

396 A great advantage of using the DRSK RCT is the possibility to modify the root canal 397 morphology as required by tutors. It can be modified to represent certain difficult situations 398 encountered during root canal treatment. By working on the customized DRSK RCT, the 399 students can get equipped to handle difficult cases and learn to manage unfamiliar situations. 400 Further studies are needed to investigate the usefulness of the DRSK RCT by evaluating the 401 outcome of treating the first patient after practicing on the DRSK RCT and comparing the 402 results with those for a control group without prior experience of working on the DRSK RCT.

\section{Conclusion}

405 The DRSK RCT can be used as an alternative to the old-school extracted teeth or as an 406 additional tool for improving dental education. Specifically, the good contrast on radiographs 407 and the realistic and freely modifiable anatomy of root canals makes the DRSK RCT a desirable 408 teaching aid. Furthermore, during COVID-19 pandemic these tooth models could help to ensure 409 teaching endodontics when patient treatment is not possible. 


\section{Abbreviations}

412 COVID-19 pandemic: coronavirus disease pandemic

413 DRSK RCT: name of the tooth model presented in this study

414 MB2: second mesiobuccal root canal

\section{Declarations}

\section{Ethics approval and consent to participate}

418 Ethics approval and consent to participate: The study was performed in the dental faculty of

419 RWTH Aachen University in Germany, after getting approval of the internal ethics committee.

420 The study was conducted in accordance with the ethical standards established in the 1964

421 Declaration of Helsinki and its subsequent amendments. All participants voluntarily took part 422 in this study and written informed consent was obtained from each participant. The 423 participating students had at all time the opportunity to withdraw their approval without 424 mentioning any reasons.

Consent for publication

427 Not applicable.

Availability of data and materials

430 All data are available from the corresponding author on reasonable request.

\section{Competing interests}

433 The authors declare that there were no competing interests. 


\section{Funding}

436 None.

437 Authors' contributions

438 All authors contributed to the study conception and design. Material preparation, data collection 439 and analysis were performed by SYM and CF. The first draft of the manuscript was written by 440 SYM. All authors (SYM, CF, AH) edited and commented on previous versions of the 441 manuscript. All authors (SYM, CF, AH) read and approved the final manuscript. 
1. De Moor R, Hulsmann M, Kirkevang LL, Tanalp J, Whitworth J. Undergraduate curriculum guidelines for endodontology. International Endodontic Journal 2013;46(12):1105-1114.

2. Al Raisi H, Dummer PMH, Vianna ME. How is Endodontics taught? A survey to evaluate undergraduate endodontic teaching in dental schools within the United Kingdom. International Endodontic Journal 2019;52(7):1077-1085.

3. Al-Anesi MS, AlKhawlani MM, Alkheraif AA, Al-Basmi AA, Alhajj MN. An audit of root canal filling quality performed by undergraduate pre-clinical dental students, Yemen. BMC Med Educ 2019;19(1):350.

4. Ribeiro DM, Reus JC, Felippe WT, Pacheco-Pereira C, Dutra KL, Santos JN, Porporatti AL, De Luca Canto G. Technical quality of root canal treatment performed by undergraduate students using hand instrumentation: a meta-analysis. International Endodontic Journal 2018;51(3):269-283.

5. Fong W, Heidarifar O, Killough S, Lappin MJ, El Karim IA. An audit on technical quality of root fillings performed by undergraduate students. International Endodontic Journal 2018;51 Suppl 3:e197-e203.

6. Fong W, Heidarifar O, Killough S, Lappin MJ, El Karim IA. An audit on technical quality of root fillings performed by undergraduate students. Int Endod J 2018;51 Suppl 3:e197-e203.

7. Polyzos NK, Sarris KG, Pita Al, Mikrogeorgis GV, Lyroudia KM. Quality of Root Canal Fillings Performed by Undergraduate Students and the Related Factors on the Treatment Outcome: A 2- to 5-Year Follow-Up. Eur Endod J 2018;3(3):179-185.

8. Saatchi M, Mohammadi G, Vali Sichani A, Moshkforoush S. Technical Quality of Root Canal Treatment Performed by Undergraduate Clinical Students of Isfahan Dental School. Iran Endod J 2018;13(1):88-93.

9. Baaij A, Ozok AR. Method of teaching undergraduate students to perform root canal treatment: It's influence on the quality of root fillings. Eur J Dent Educ 2018;22(2):e221-e227.

10. Dominici JT, Eleazer PD, Clark SJ, Staat RH, Scheetz JP. Disinfection/sterilization of extracted teeth for dental student use. Journal of Dental Education 2001;65(11):12781280.

11. Hope CK, Griffiths DA, Prior DM. Finding an alternative to formalin for sterilization of extracted teeth for teaching purposes. Journal of Dental Education 2013;77(1):68-71.

12. Qualtrough AJ. Undergraduate endodontic education: what are the challenges? British Dental Journal 2014;216(6):361-364.

13. Schlenz MA, Schmidt A, Wostmann B, Kramer N, Schulz-Weidner N. Students' and lecturers' perspective on the implementation of online learning in dental education due to SARS-CoV-2 (COVID-19): a cross-sectional study. BMC Med Educ 2020;20(1):354.

14. Haroon Z, Azad AA, Sharif M, Aslam A, Arshad K, Rafiq S. COVID-19 Era: Challenges and Solutions in Dental Education. J Coll Physicians Surg Pak 2020;30(10):129-131.

15. Lieberman JA, Nester T, Emrich B, Staley EM, Bourassa LA, Tsang HC. Coping With COVID-19. Am J Clin Pathol 2020.

16. Spenst A, Kahn $\mathrm{H}$. The use of a plastic block for teaching root canal instrumentation and obturation. Journal of Endodontics 1979;5(9):282-284. 
17. Boutsioukis $C$, Verhaagen $B$, Walmsley AD, Versluis $M$, van der Sluis LW. Measurement and visualization of file-to-wall contact during ultrasonically activated irrigation in simulated canals. International Endodontic Journal 2013;46(11):1046-1055.

18. Bitter K, Gruner D, Wolf O, Schwendicke F. Artificial Versus Natural Teeth for Preclinical Endodontic Training: A Randomized Controlled Trial. Journal Endodontics 2016;42(8):1212-1217.

19. Al-Sudani DI, Basudan SO. Students' perceptions of pre-clinical endodontic training with artificial teeth compared to extracted human teeth. European Journal of Dental Education 2017;21(4):e72-e75.

20. dos S. Luz D, de S. Ourique F, Scarparo RK, Vier-Pelisser FV, Morgental RD, Waltrick SB, de Figueiredo JA. Preparation time and perceptions of Brazilian specialists and dental students regarding simulated root canals for endodontic teaching: a preliminary study. Journal of Dental Education 2015;79(1):56-63.

21. Robberecht L, Chai F, Dehurtevent M, Marchandise P, Becavin T, Hornez JC, Deveaux E. A novel anatomical ceramic root canal simulator for endodontic training. European Journal of Dental Education 2017;21(4):e1-e6.

22. Reymus M, Fotiadou C, Kessler A, Heck K, Hickel R, Diegritz C. 3D printed replicas for endodontic education. International Endodontic Journal 2019;52(1):123-130.

23. Martins JNR, Marques D, Silva E, Carames J, Mata A, Versiani MA. Second mesiobuccal root canal in maxillary molars-A systematic review and meta-analysis of prevalence studies using cone beam computed tomography. Archives of Oral Biology 2019:104589.

24. Nassri MR, Carlik J, da Silva CR, Okagawa RE, Lin S. Critical analysis of artificial teeth for endodontic teaching. Journal of Applied Oral Science 2008;16(1):43-49.

25. Tchorz JP, Ganter PA, Woelber JP, Stampf S, Hellwig E, Altenburger MJ. Evaluation of an improved endodontic teaching model: do preclinical exercises have an influence on the technical quality of root canal treatments? International Endodontic Journal 2014;47(5):410-415.

26. Gancedo-Caravia L, Bascones J, Garcia-Barbero E, Arias A. Suitability of different tooth replicas for endodontic training: perceptions and detection of common errors in the performance of postgraduate students. International Endodontic Journal 2019.

27. Hasselgren G, Tronstad L. The use of transparent teeth in the teaching of preclinical endodontics. Journal Endodontics 1975;1(8):278-280.

28. Iyer P, Aziz K, Ojcius DM. Impact of COVID-19 on dental education in the United States. J Dent Educ 2020;84(6):718-722. 


\section{Figure Legends}

Figure 1. Radiographs showing treatment errors done in student courses (a, c, e). Graphics of

529 the endodontic tooth models that were developed according to the recorded errors (b, d, f).

530 a) Tooth 12 with j-shape root canal and a via falsa; b) Premolar tooth model with an apically

531 strongly curved root canal (draft + produced model); c) Tooth 45 with a Type II shaped root

532 canal and an incomplete obturated root canal; d) Incisor tooth model with a Type II shaped root

533 canal (draft + produced model); e) Tooth 36 with a perforation of the pulp ground that was

534 assumed to be a further root canal by students; $\mathrm{f}$ ) Molar tooth model with a hidden mb2 (draft

$535+$ produced model).

536

537 Figure 2. Picture of a DRSK RCT model of a maxillary first molar with completed root filling with gutta-percha points performed by a student with lateral condensation technique.

540 Figure 3. Radiographs of a maxillary first molar DRSK RCT tooth model: a) lengths measuring 541 with silverpoints b) control of gutta-percha master cones c) control of the completed root canal 542 filling

544 Figure 4. Bar chart showing the mean opinions of students on helpfulness of tooth model in the

545 first investigation (grey) and the second one after modification (black).

546 Line: standard deviation/ asterisk: $\mathrm{p}<0.05$ 
548 Figure 5. Bar chart showing the mean opinions of dental instructors on helpfulness of tooth

549 model in the first investigation (grey) and the second one after modification (black).

550 Line: standard deviation/ asterisk: $\mathrm{p}<0.05$ 


\section{Figures}

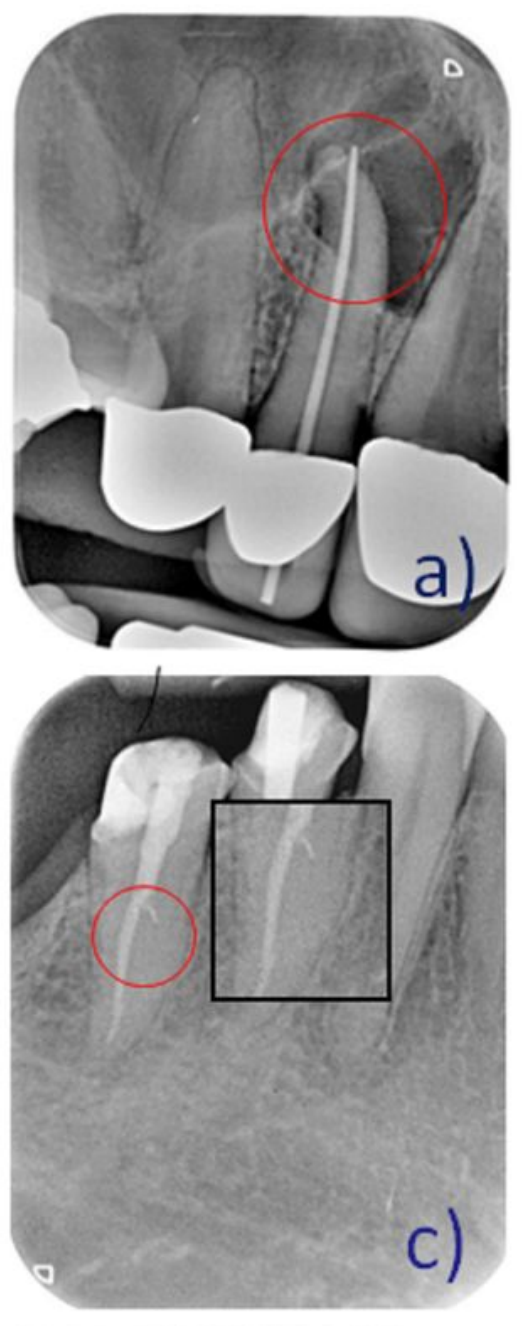

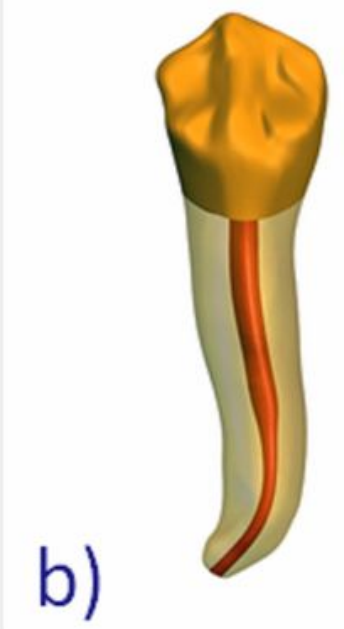
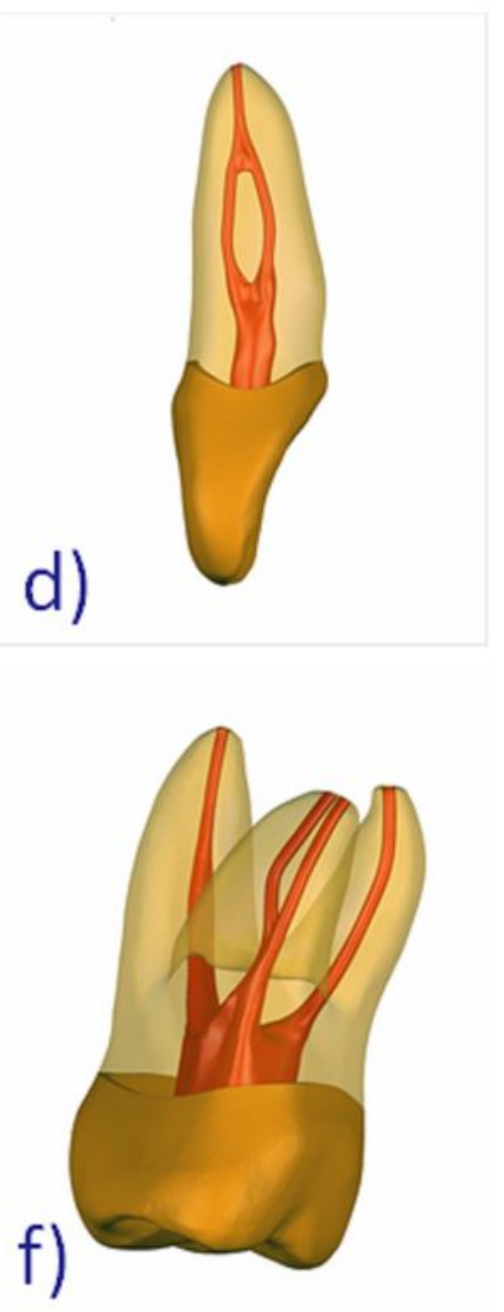
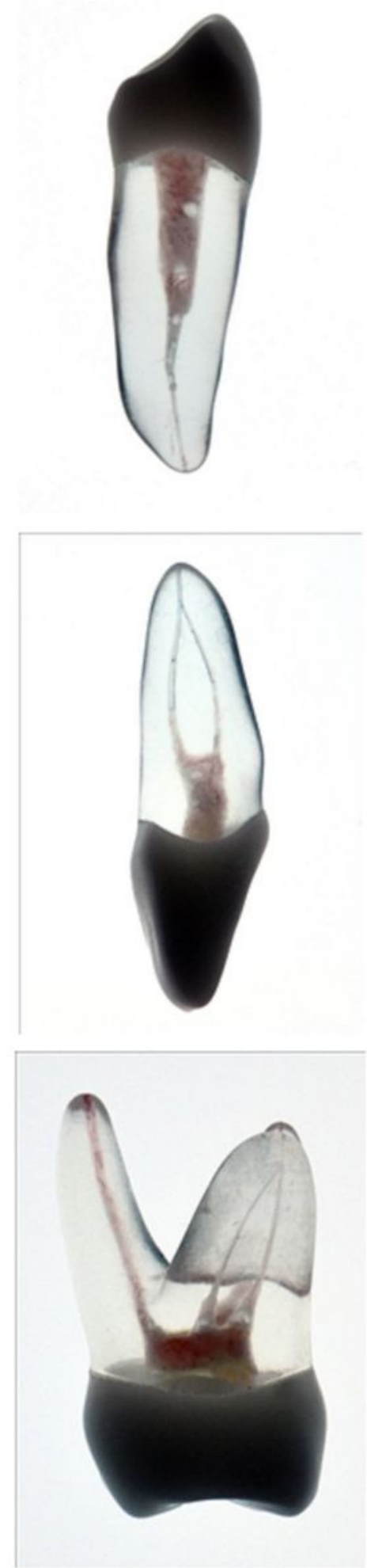

\section{Figure 1}

Radiographs showing treatment errors done in student courses (a, c, e). Graphics of the endodontic tooth models that were developed according to the recorded errors $(b, d, f)$. a) Tooth 12 with $j$-shape root canal and a via falsa; b) Premolar tooth model with an apically strongly curved root canal (draft + produced 
model); c) Tooth 45 with a Type II shaped root canal and an incomplete obturated root canal; d) Incisor tooth model with a Type II shaped root canal (draft + produced model); e) Tooth 36 with a perforation of the pulp ground that was assumed to be a further root canal by students; f) Molar tooth model with a hidden mb2 (draft + produced model).

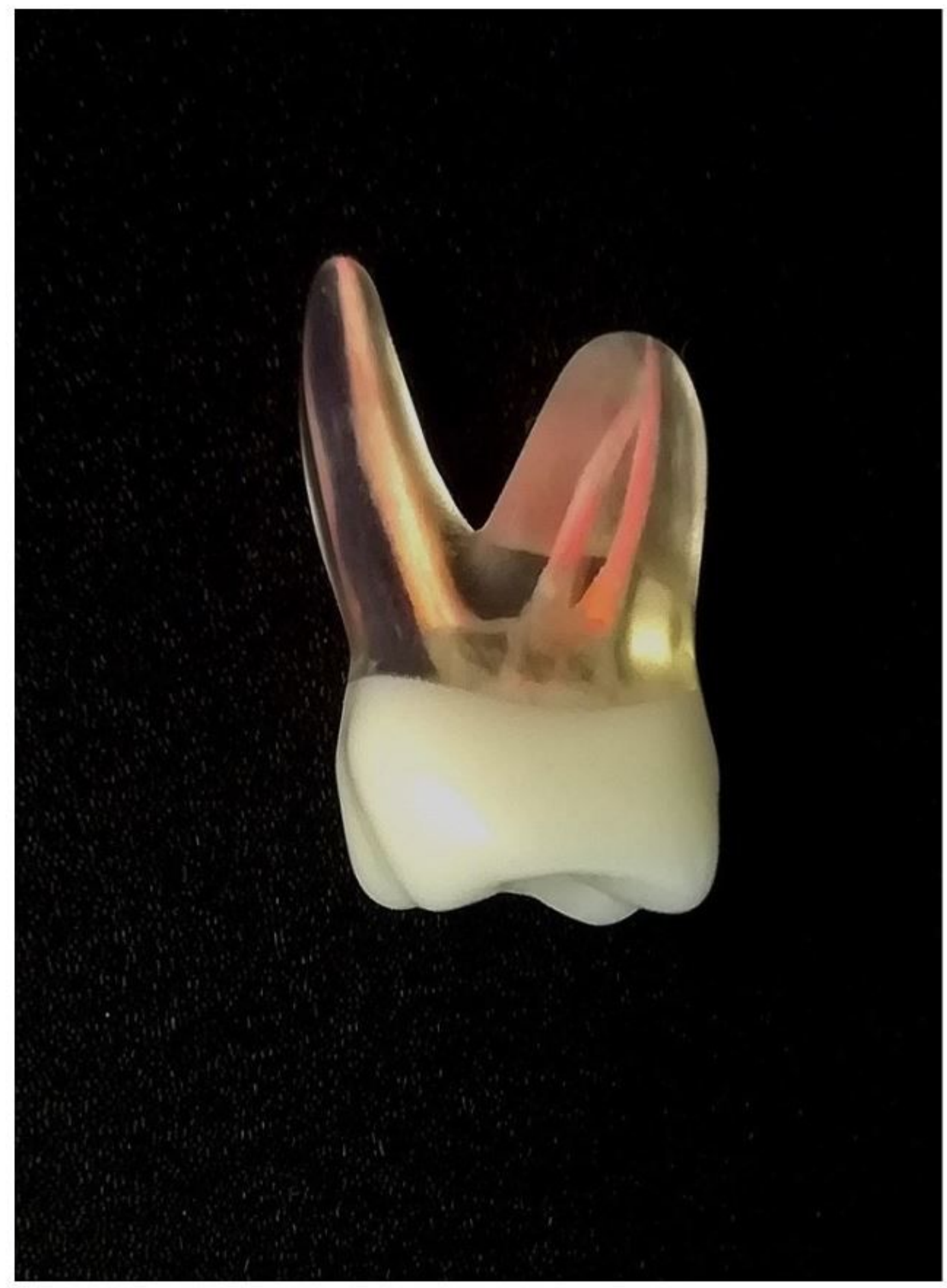

Figure 2 
Picture of a DRSK RCT model of a maxillary first molar with completed root filling with gutta-percha points performed by a student with lateral condensation technique.

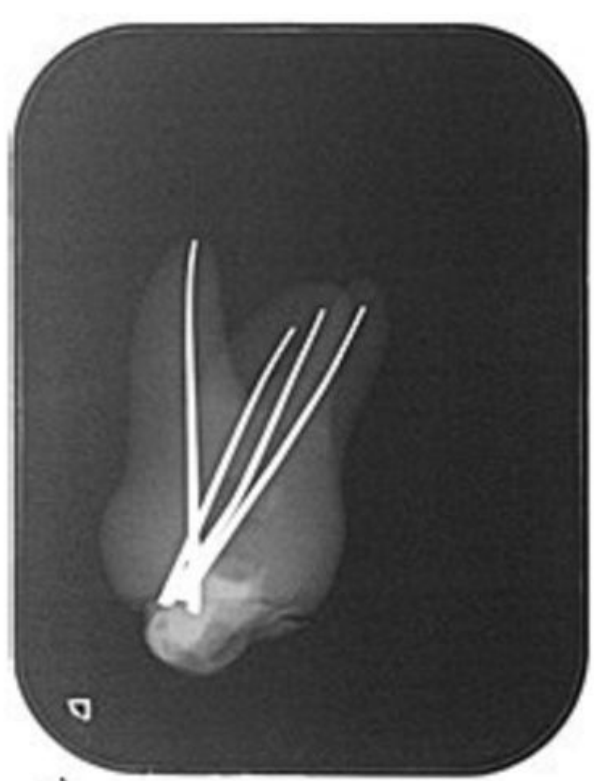

a)

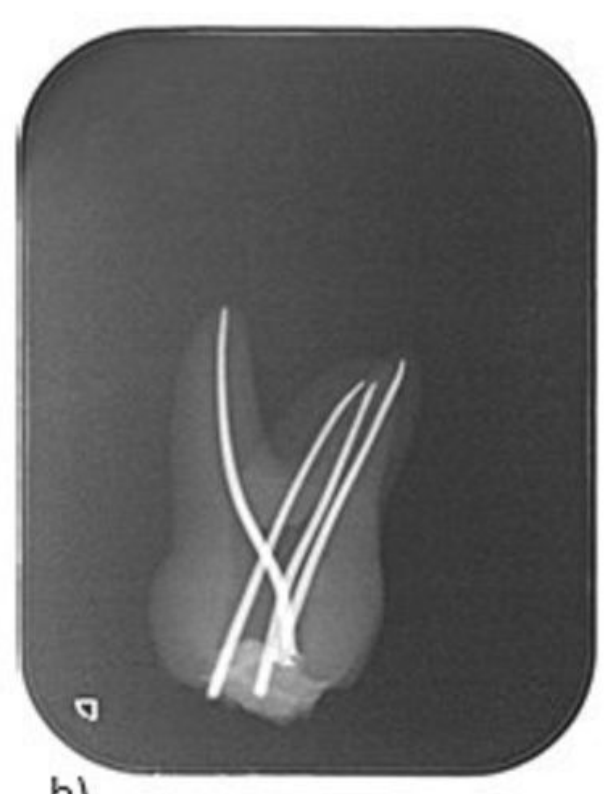

b)

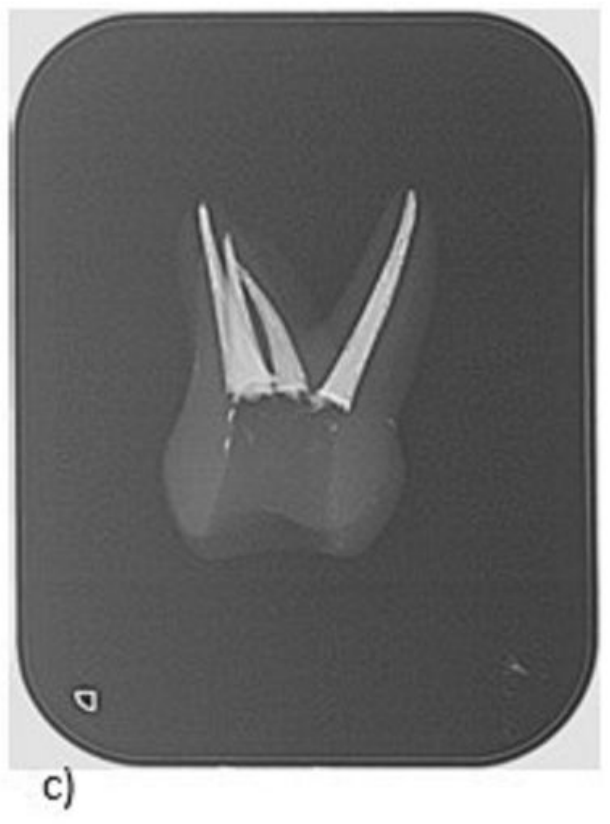

c)

\section{Figure 3}

Radiographs of a maxillary first molar DRSK RCT tooth model: a) lengths measuring with silverpoints $b$ ) control of gutta-percha master cones $\mathrm{c}$ ) control of the completed root canal filling 


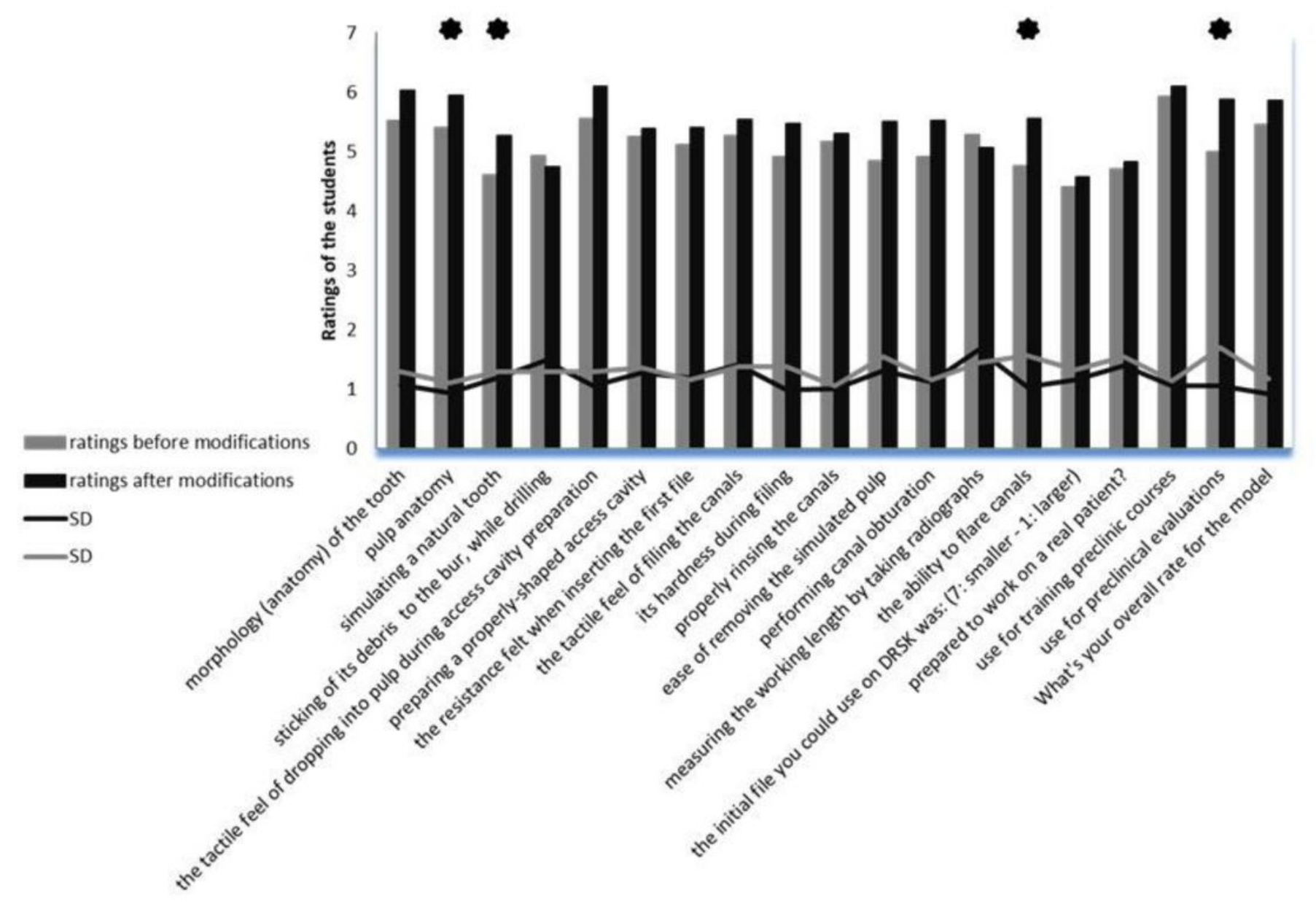

Figure 4

Bar chart showing the mean opinions of students on helpfulness of tooth model in the first investigation (grey) and the second one after modification (black). Line: standard deviation/ asterisk: $p<0.05$ 


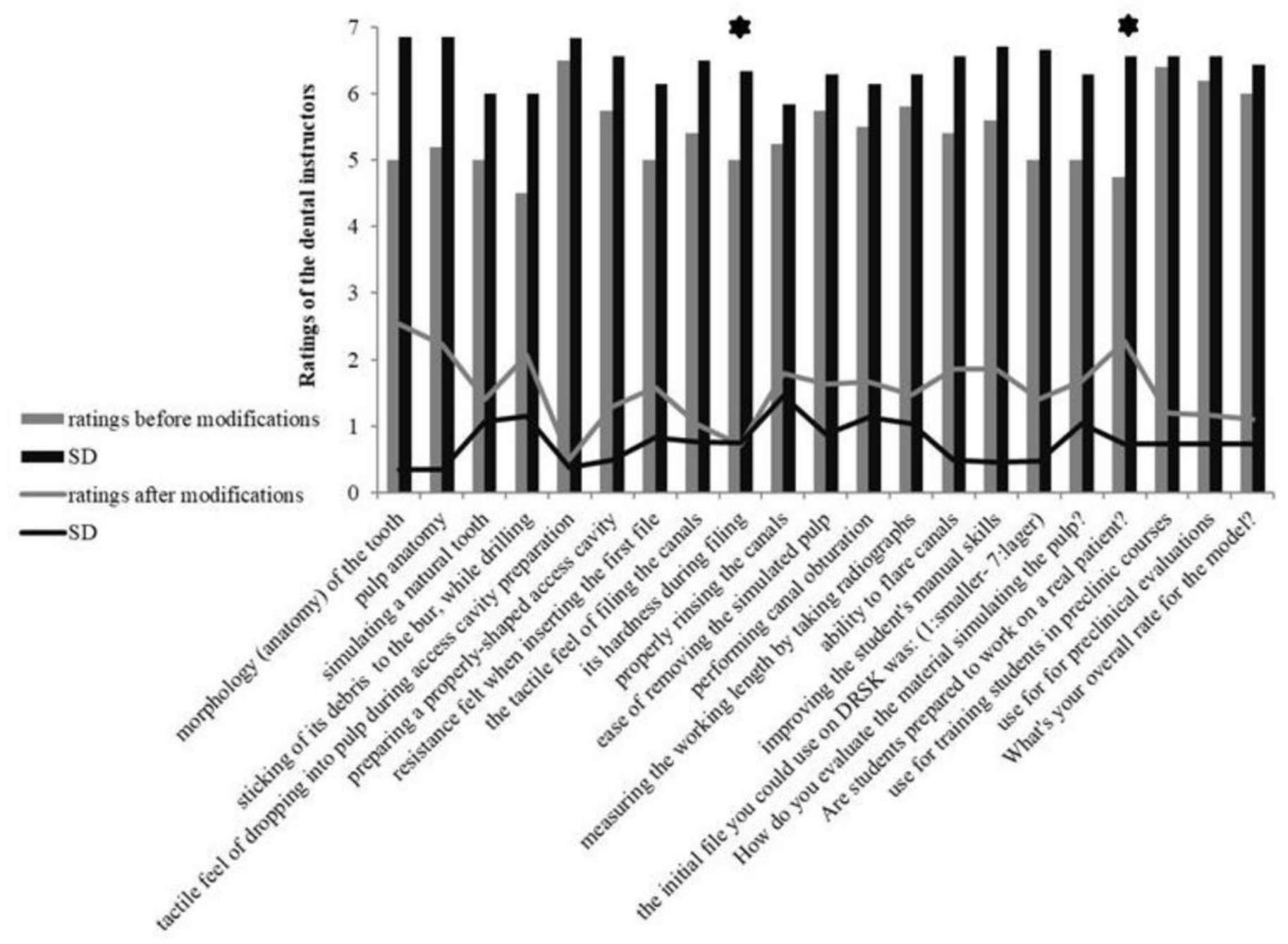

Figure 5

Bar chart showing the mean opinions of dental instructors on helpfulness of tooth model in the first investigation (grey) and the second one after modification (black). Line: standard deviation/ asterisk: $p<$ 0.05 